\title{
Nuclear cardiology at the door of a new era: better to save $\mathrm{mSv}$ or to reduce imaging time?
}

\author{
Assuero Giorgetti • Dario Genovesi • Paolo Marzullo
}

Published online: 5 September 2012

(C) Springer-Verlag 2012

\section{Nuclear cardiology in the last 30 years}

The evolution of nuclear cardiology has gone through several clinical protocols that can be summarized as ${ }^{201} \mathrm{Tl}$ stress/redistribution, ${ }^{201} \mathrm{Tl}$ stress/redistribution with delayed imaging, ${ }^{201} \mathrm{Tl}$ stress/redistribution with reinjection, singleor double-day ${ }^{99 \mathrm{~m}} \mathrm{Tc}$-labelled radiotracers, and ${ }^{201} \mathrm{Tl} /{ }^{99 \mathrm{~m}} \mathrm{Tc}$ combined approaches. This evolution covered more or less 30 years with a significant (at least $25 \%$ ) increase in the accuracy of the method, but (up to few years ago) a decrease in the $\mathrm{mSv}$ to the patient of less than $5 \%$. Recent advances in hardware and software applied to nuclear cardiology [1] have led to short imaging times and/or low-dose studies, but the choice of the best combination is still difficult.

Nuclear cardiology at the time of the "Ulysses syndrome"

Recently, some cardiologists have raised the concern that marketing messages, high patient demand and defensive medicine may lead to the vicious circle of the "Ulysses syndrome" [2] in which Mr. Ulysses is advised to have a cardiological check-up after 10 years of war. After a long journey across imaging laboratories, he has a lot of expensive cardiological examinations and with a radiation dose from more than 4,000 chest radiographs. Ulysses is tired of useless examinations, exorbitant costs and unacceptable risks. Indeed, the number of radiological and nuclear medicine examinations has been growing rapidly in recent years. In the US, the number of myocardial perfusion imaging studies has grown from less than 3 million to 10 million per year over a period of 12 years (from 1990 to 2002) [3].

\footnotetext{
A. Giorgetti $(\bowtie) \cdot$ D. Genovesi $\cdot$ P. Marzullo

Fondazione CNR/Regione Toscana "Gabriele Monasterio", Pisa, Italy

e-mail: asso@ftgm.it
}

The growth in medical "ionizing" procedures has raised the concern of the association between the increase in radiation exposure, even at the low levels used, and an augmented risk of cancer. From a single diagnostic procedure, such risk is surely low but, on a population basis, cancers become more likely as the number of procedures performed increases. These notions are clear and well known in the radiological and nuclear medicine communities but, apparently, they are not so clearly recognized in the cardiological and internal medicine communities.

\section{Exploiting the advantages of the emerging technology}

Reducing imaging time and saving money

The availability of new technologies initially started a race toward superfast imaging, maintaining the dose more or less unchanged when compared to standard myocardial scintigraphy. In spite of differences in hardware and software, the new cameras and software are all associated with a dramatic increase in count statistics, and spatial and energy resolution, and have been used clinically to reduce the overall duration of myocardial perfusion scintigraphy. The studies obtained with the emerging technology have been validated by comparison with studies performed with the same injected dose during the same patient visit on a conventional dual head SPECT camera. As demonstrated by several investigators, the emerging technology has allowed acquisition times for a myocardial perfusion scan to be reduced to between half the conventional time and a minimum of two minutes [4]. This "fast and furious" nuclear cardiology imaging is now a reality that has led to improvements in patient compliance with the studies, increase in the efficiency of nuclear laboratories, and reductions in motion artefacts and costs. 
Reducing dose and radiation burden to the patient

The use of new cameras and related software has led to dramatic increases in system sensitivity and in myocardial counts detected. Unfortunately, it is not easy to prospectively assess the lowest achievable imaging dose because we need to be conservative in such a study and avoid a scan that is inadequate for clinical interpretation. The new technology permits list-mode acquisition so that it is possible to simulate a scan with an arbitrary activity retrospectively from original full-dose acquisition data. Using this approach, Herzog et al. [5] showed that a 2-min acquisition time is the lower limit at which images quality is preserved for the GE Healthcare NM530c scanner, and we could then suggest a dose reduction to half the standard one. In a recent study, Duvall et al. [6] studied 131 patients using CZT Alcyone Technology and ${ }^{99 \mathrm{~m}} \mathrm{Tc}$-sestamibi $(5 \mathrm{mCi}$ rest dose, $15 \mathrm{mCi}$ stress dose). When compared to a conventional SPECT camera in 27 patients, total rest and stress perfusion deficits and calculated LVEF results were similar. The effective dose was $49.2 \%$ less than with conventional ${ }^{99 \mathrm{~m}} \mathrm{Tc}$ studies and $75.7 \%$ less than with conventional ${ }^{201} \mathrm{Tl} /{ }^{99 \mathrm{~m}} \mathrm{Tc}$ dual isotope studies. Similarly, in a study in our institution [7], we investigated 137 consecutive patients with known or suspected coronary artery disease using ultrafast SPECT perfusion imaging and invasive coronary angiography as the gold standard. The results demonstrated that a single-day stress/ rest low-dose ultrafast protocol with a CZT camera is clinically feasible, provides high image quality and high diagnostic accuracy when compared to coronary angiography. We also obtained similar results using a low-dose protocol in a population of obese patients [8].

In another study, the patient dose was reduced to $4.3 \mathrm{mSv}$ for stress/rest protocols using a background activity subtraction algorithm [9]. The comparison between standard dose and low-dose background subtracted protocols demonstrated clinical agreement in $98 \%$ of patients, confirming the possibility of significantly reducing radiation exposure while maintaining the diagnostic power of myocardial scintigraphy.

In addition, the radiation burden to the patient can be reduced by performing a stress-only protocol. If appropriateness criteria for myocardial perfusion imaging are accurately respected, more than $50 \%$ of stress studies can theoretically be expected to be normal. In a recent study by Nakazato et al. [10], the diagnostic accuracy of a CZT camera with automated quantification of the total perfusion defect was tested versus invasive coronary angiography. With the combined upright-supine approach, the sensitivity for detection of significant coronary artery disease was $94 \%$ and the specificity was $86 \%$. Although limited by the size of the sample of patients studied, this study indicates the potential of the new technology which could permit very low-dose $(<2.5 \mathrm{mSv})$ ultrafast (4-min upright scan+4-min supine scan) myocardial perfusion scintigraphy.

Considering these results, nuclear cardiology remains an attractive modality for incorporation into multimodality or hybrid imaging systems. Recently, Pazhenkottil et al. described a novel dose-saving fast-track hybrid algorithm [11], using CT coronary angiography first, followed by stressonly low-dose myocardial scintigraphy (using Evolution for cardiology software) if a coronary stenosis of $\geq 50 \%$ diameter narrowing or equivocal findings were observed. Only abnormal stress perfusion scans were followed by rest perfusion scintigraphy. The mean effective radiation dose was significantly lower for the individualized $(4.8 \pm 3.4 \mathrm{mSv})$ than for the comprehensive method $(8.1 \pm 1.5 \mathrm{mSv})$, resulting in a significant reduction in the total population radiation dose.

\section{Conclusions: “journey home to Ithaca”}

Advances in technology are a challenge for modern medicine. Overuse, inappropriate use or underuse of this technology are of equal concern and the issue is not intrinsic to the technology used but to the way the technology is used. It is conceivable that, in the future, aggressive clinical dosereduction algorithms can be customized to the patient age, since radiation risk models assume that risk is inversely proportional to patient age. In this light, new protocols are to be explored to further reduce radiation dose to the patient rather than to save time in acquisitions of less than $5 \mathrm{~min}$. Available and future results should be clearly disseminated to enhance the appeal of nuclear cardiology to the cardiological community.

\section{References}

1. Garcia EV, Faber TL, Esteves FP. Cardiac dedicated ultrafast SPECT cameras: new designs and clinical implications. J Nucl Med. 2011;52:210-7.

2. Picano E. The risks of inappropriateness in cardiac imaging. Int $J$ Environ Res Public Health. 2009;6:1649-64.

3. IMV. 2003 nuclear medicine census market summary report. Des Plaines, IL: IMV Medical Information Division.

4. Slomka PJ, Patton JA, Berman DS, Germano G. Advances in technical aspects of myocardial perfusion SPECT imaging. J Nucl Cardiol. 2009;16:255-76.

5. Herzog BA, Buechel RR, Katz R, Pazhenkottil A, Valenta I, Gaemperli $\mathrm{O}$, et al. Nuclear myocardial perfusion imaging with a cadmium-zinc-telluride detector technique: optimized protocol for scan time reduction. J Nucl Med. 2010;51:46-51.

6. Duvall WL, Croft LB, Ginsberg ES, Einstein AJ, Guma KA, George $\mathrm{T}$, et al. Reduced isotope dose and imaging time with a highefficiency CZT SPECT camera. J Nucl Cardiol. 2011;18:847-57.

7. Gimelli A, Bottai M, Genovesi D, Giorgetti A, Di Martino F, Marzullo P. High diagnostic accuracy of low-dose gated-SPECT 
with solid-state ultrafast detectors: preliminary clinical results. Eur J Nucl Med Mol Imaging. 2012;39(1):83-90.

8. Gimelli A, Bottai M, Giorgetti A, Genovesi D, Filidei E, Marzullo P. Evaluation of ischaemia in obese patients: feasibility and accuracy of a low-dose protocol with a cadmium-zinc telluride camera. Eur J Nucl Med Mol Imaging. 2012;39(8):1254-61.

9. Nkoulou R, Pazhenkottil AP, Kuest SM, Ghadri JR, Wolfrum M, Husmann L, et al. Semiconductor detectors allow low-dose-lowdose 1-day SPECT myocardial perfusion imaging. J Nucl Med. 2011;52(8):1204-9.
10. Nakazato R, Tamarappoo BK, Kang X, Wolak A, Kite F, Hayes SW, et al. Quantitative upright-supine high-speed SPECT myocardial perfusion imaging for detection of coronary artery disease: correlation with invasive coronary angiography. J Nucl Med. 2010;51(11):1724-31.

11. Pazhenkottil AP, Herzog BA, Husmann L, Buechel RR, Burger IA, Valenta I, et al. Non-invasive assessment of coronary artery disease with CT coronary angiography and SPECT: a novel dose-saving fast-track algorithm. Eur J Nucl Med Mol Imaging. 2010;37:522-7. 